\title{
On the Support of Solutions to a Two-Dimensional Nonlinear Wave Equation
}

\author{
Wenbin Zhang, ${ }^{1}$ Jiangbo Zhou, ${ }^{2}$ Lixin Tian, ${ }^{2}$ and Sunil Kumar ${ }^{3}$ \\ ${ }^{1}$ Taizhou Institute of Science and Technology, NUST, Taizhou, Jiangsu 225300, China \\ ${ }^{2}$ Nonlinear Scientific Research Center, Faculty of Science, Jiangsu University, Zhenjiang, Jiangsu 212013, China \\ ${ }^{3}$ Department of Mathematics, National Institute of Technology, Jamshedpur, Jharkhand 831014, India
}

Correspondence should be addressed to Jiangbo Zhou; zhoujiangbo@yahoo.cn

Received 10 January 2013; Accepted 21 January 2013

Academic Editor: Ji Gao

Copyright (C) 2013 Wenbin Zhang et al. This is an open access article distributed under the Creative Commons Attribution License, which permits unrestricted use, distribution, and reproduction in any medium, provided the original work is properly cited.

It is shown that if $u$ is a sufficiently smooth solution to a two-dimensional nonlinear wave equation such that there exists $L>0$ with supp $u(i) \subseteq[-L, L] \times[-L, L]$, for $i=0,1$, then $u \equiv 0$.

\section{Introduction}

In this paper, we consider the following two-dimensional nonlinear wave equation:

$$
u_{t}+a u u_{x}+b u_{x x x}+c u_{y y x}+d u_{x}=0
$$

where $a, b, c, d$ are arbitrary positive constants. Equation (1) was recently derived by Gottwald [1] for large scale motion from the barotropic quasigeostrophic equation as a twodimensional model for Rossby waves. He [2] showed that (1) has traveling wave solutions via the homotopy perturbation method. Using a subequation method, the traveling wave solutions are also studied by Fu et al. [3]. Aslan [4] constructed solitary wave solutions and periodic wave solutions to (1) by the Exp-function method.

For $a=b=c=1$ and $d=0$ in (1), one obtains the classical Zakharov-Kuznetsov (ZK) equation [5], which is a mathematical model to describe the propagation of nonlinear ion-acoustic waves in magnetized plasma. Solitary wave solutions and the Cauchy problem to ZK equation have extensively been studied in the literature ([6-11]). Panthee [12] proved that if a sufficiently smooth solution to the initial value problem associated with the $\mathrm{ZK}$ equation is supported compactly in a nontrivial time interval, then it vanishes identically. Recently, Bustamante et al. [13] showed that sufficiently smooth solutions of the $\mathrm{ZK}$ equation that have compact support for two different times are identically zero.

The purpose of this paper is to investigate the support of solutions to (1). To solve the problem, we mainly use the ideas of [12-15]. The main result is as follows.

Theorem 1. Assume that $b>c$ and $L>0$, if $u \in C([0,1]$; $\left.H^{4}\left(R^{2}\right)\right) \cap C^{1}\left([0,1] ; L^{2}\left(R^{2}\right)\right)$ is a solution of $(1)$ such that

$$
\text { supp } u(0), \text { supp } u(1) \subseteq[-L, L] \times[-L, L] \text {; }
$$

then, $u \equiv 0$.

\section{Preliminary Estimates}

Lemma 2 (see [13]). Assume that $s>0$ and $\kappa>0$. (i) If $f \in$ $H^{s}\left(R^{2}\right) \cap L^{2}\left(e^{2 \kappa x} d x d y\right)$, then

$$
\left\|J^{\theta s}\left(e^{(1-\theta) \kappa x} f\right)\right\|_{L^{2}} \leq C\left\|J^{s} f\right\|_{L^{2}}^{\theta}\left\|e^{\kappa x} f\right\|_{L^{2}}^{1-\theta}
$$

(ii) if $f \in H^{s}\left(R^{2}\right) \cap L^{2}\left(e^{2(\kappa x+\kappa y)} d x d y\right)$, then

$$
\left\|J^{\theta s}\left(e^{(1-\theta)(\kappa x+\kappa y)} f\right)\right\|_{L^{2}} \leq C\left\|J^{s} f\right\|_{L^{2}}^{\theta}\left\|e^{\kappa x+\kappa y} f\right\|_{L^{2}}^{1-\theta},
$$

where $\theta \in[0,1], C=C(s, \kappa)$, and $\left[J^{s} f\right]^{\wedge}(\xi):=\left(1+|\xi|^{2}\right)^{s / 2}$ $[f]^{\wedge}(\xi)$. 
Lemma 3. Assume that $\kappa>0$, if $u \in C\left([0,1] ; H^{4}\left(R^{2}\right)\right) \cap$ $C^{1}\left([0,1] ; L^{2}\left(R^{2}\right)\right)$ is a solution of (1) such that $u(0) \in$ $L^{2}\left(e^{2 \kappa x} d x d y\right)$; then, $u(t)$ is bounded in $H^{3}\left(e^{2 \kappa x} d x d y\right)$.

Proof. Assume that $\varphi(x) \in C^{\infty}(R)$ is a decreasing function with $\varphi(x)=1$ if $x<1$ and $\varphi(x)=0$ if $x>10$. Let $\theta_{n}(x)=$ $\int_{0}^{x} \varphi(t / n) d t$ for $n \in Z^{+}$and $\phi_{n}(x)=e^{2 k \theta_{n}(x)}$. It is easy to check that $\phi_{n}(x) \leq \phi_{n+1}(x)$ and

$$
\left|\phi_{n}^{(j)}(x)\right| \leq C_{j, k} \phi_{n}(x), \quad \forall j \in Z^{+}, \forall x \in R
$$

Multiplying (1) by $u \phi_{n}$ and integrating by parts in $R_{x y}^{2}$, we obtain

$$
\begin{aligned}
\frac{1}{2} \frac{d}{d t} \int u^{2} \phi_{n}= & \frac{a}{3} \int u^{3} \phi^{\prime}-\frac{3 b}{2} \int\left(u_{x}\right)^{2} \phi_{n}^{\prime}+\frac{b}{2} \int u^{2} \phi_{n}^{\prime \prime \prime} \\
& -\frac{c}{2} \int\left(u_{y}\right)^{2} \phi^{\prime}+\frac{d}{2} \int u^{2} \phi_{n}^{\prime} \\
\leq & \frac{a}{3}\|u\|_{L^{\infty}\left(R^{2}\right)} C_{1, \kappa} \int u^{2} \phi_{n}+\frac{b}{2} C_{3, \kappa} \int u^{2} \phi_{n} \\
& +\frac{d}{2} C_{1, \kappa} \int u^{2} \phi_{n} \\
\leq & \left(\frac{a}{3} C\|u\|_{C\left([0,1] ; H^{2}\right)}+\frac{b}{2} C_{3, k}+\frac{d}{2} C_{1, k}\right) \int u^{2} \phi_{n} \\
= & C_{k, u} \int u^{2} \phi_{n} .
\end{aligned}
$$

Applying Gronwall Lemma and the Monotone Convergence Theorem, we have

$$
\int u(t)^{2} e^{2 \kappa x} d x d y \leq C \int u(0)^{2} e^{2 \kappa x} d x d y, \quad \forall t \in[0,1]
$$

This proves that $u(t)$ is bounded in $L^{2}\left(e^{2 \kappa x} d x d y\right)$.

Applying Lemma 2 with $s=4$ and $\theta=3 / 4$, we have that $u(t)$ is bounded in $H^{3}\left(e^{2 \kappa x} d x d y\right)$. Here, we used the fact that $u \in C\left([0,1] ; H^{4}\right)$. This completes the proof of the lemma.

Lemma 4. Assume that $\kappa>0, b>c$, and $u \in C([0,1]$; $\left.H^{4}\left(R^{2}\right)\right) \cap C^{1}\left([0,1] ; L^{2}\left(R^{2}\right)\right)$ is a solution of (1). (i) If $u(0) \in L^{2}\left(e^{2 \kappa x} e^{2 \kappa|y|} d x d y\right)$, then $u(t)$ is bounded in $H^{3}\left(e^{2 \kappa x} e^{2 \kappa|y|} d x d y\right)$; (ii) if $u(1) \in L^{2}\left(e^{-2 k x} e^{2 k|y|} d x d y\right)$, then $u(t)$ is bounded in $H^{3}\left(e^{-2 \kappa x} e^{2 \kappa|y|} d x d y\right)$.

Proof. Letting $v(t)=e^{\kappa x} u(t)$ and $u$ a solution to (1), we have

$$
\begin{aligned}
e^{k x} u^{\prime}= & \kappa\left(a u+b \kappa^{2}+d\right) v-\left(a u+3 b \kappa^{2}+d\right) v_{x}+3 b \kappa v_{x x} \\
& -b v_{x x x}+c \kappa v_{y y}-c v_{y y x} .
\end{aligned}
$$

Multiplying (8) by $v \phi_{n}(y)$ and integrating by parts in $R_{x y}^{2}$, we obtain

$$
\begin{aligned}
\int e^{\kappa x} u^{\prime} v \phi_{n}= & a \kappa \int u v^{2} \phi_{n}+\frac{a}{2} \int v^{2} u_{x} \phi_{n}+\left(b \kappa^{3}+d \kappa\right) \int v^{2} \phi_{n} \\
& -3 b \kappa \int\left(v_{x}\right)^{2} \phi_{n}-c \kappa \int\left(v_{y}\right)^{2} \phi_{n}+\frac{c}{2} \kappa \int v^{2} \phi_{n}^{\prime \prime} \\
& -c \int v_{x} v_{y} \phi_{n}^{\prime} .
\end{aligned}
$$

Note that $\left|\phi_{n}^{\prime}(y)\right| \leq 2 \kappa \phi_{n}(y)$ and

$$
\int e^{k x} u^{\prime} v \phi_{n}=\frac{1}{2} \frac{d}{d t} \int v^{2} \phi_{n}, \quad \text { a.e } t \in[0,1] .
$$

It follows from (9) that

$$
\begin{aligned}
\frac{1}{2} \frac{d}{d t} \int v^{2} \phi_{n} \\
\leq a \kappa C\|u\|_{C\left([0,1] ; H^{2}\left(R^{2}\right)\right)} \int v^{2} \phi_{n} \\
\quad+a C\left\|u_{x}\right\|_{C\left([0,1] ; H^{2}\left(R^{2}\right)\right)} \int v^{2} \phi_{n} \\
\quad+\left(b \kappa^{3}+d \kappa\right) \int v^{2} \phi_{n} \\
\quad-c \kappa \int\left[\left(v_{x}\right)^{2}+2\left|v_{x}\right|\left|v_{y}\right|+\left(v_{y}\right)^{2}\right] \phi_{n} \\
\quad+\frac{c}{2} \kappa C_{2, \kappa} \int v^{2} \phi_{n} \\
=C_{\kappa, u} \int v^{2} \phi_{n}-c \kappa \int\left(\left|v_{x}\right|+\left|v_{y}\right|\right)^{2} \phi_{n} \\
\leq C_{\kappa, u} \int v^{2} \phi_{n}, \quad \text { a.e } t \in[0,1] .
\end{aligned}
$$

Since $u \in C\left([0,1] ; H^{4}\right)$ and $u(t)$ is bounded in $L^{2}\left(e^{2 \kappa x} e^{2 \kappa y} d x d y\right)$, applying Lemma 2 with $s=4$ and $\theta=3 / 4$, we have that $u(t)$ is also bounded in $H^{3}\left(e^{2 \kappa x} e^{2 \kappa y} d x d y\right)$.

Similarly, we can prove that $u(t)$ is bounded in $H^{3}\left(e^{2 \kappa x} e^{-2 \kappa y} d x d y\right)$. Let $\widetilde{u}(x, y, t)=u(x,-y, t)$; then, $\widetilde{u}$ is a solution to (1) and satisfies $\widetilde{u}(0) \in L^{2}\left(e^{2 \kappa x} e^{2 \kappa|y|} d x d y\right)$, and therefore $\tilde{u}$ is bounded in $H^{3}\left(e^{2 \kappa x} e^{2 \kappa y} d x d y\right)$. This proves (i).

Now, we prove (ii). Let $\bar{u}(x, y, t)=u(-x, y, 1-t)$; then, $\bar{u}(x, y, t)$ is also a solution of (1) and satisfies the hypothesis of (i). This proves (ii) and completes the proof of the lemma.

Remark 5. In particular, if the conditions for $u(0)$ and $u(1)$ given in (i) and (ii), respectively, are satisfied, then $u(t)$ is bounded in $H^{3}\left(e^{2 \kappa|x|} e^{2 \kappa|y|} d x d y\right)$.

Lemma 6 (see [13]). Let $\kappa>0, v \in C^{1}\left([0,1] ; L^{2}\left(R^{2}\right)\right)$ is a function such that $v(t)$ is bounded in $L^{2}\left(e^{2 \kappa|x|} e^{2 \kappa|y|} d x d y\right)$, and $v^{\prime} \in L^{1}\left([0,1] ; L^{2}\left(e^{2 \kappa|x|} e^{2 \kappa|y|} d x d y\right)\right)$. Then, for all $\lambda \in R$ and 
all $\xi=\left(\xi_{1}, \xi_{2}\right) \in R^{2}$, the functions $t \rightarrow\left[e^{\lambda x} v(t)\right]^{\wedge}(\xi)$ and $t \rightarrow\left[e^{\lambda y} v(t)\right]^{\wedge}(\xi)$ are absolutely continuous in $[0,1]$ with derivatives $\left[e^{\lambda x} v^{\prime}(t)\right]^{\wedge}(\xi)$ and $\left[e^{\lambda y} v^{\prime}(t)\right]^{\wedge}(\xi)$ a.e. $t \in[0,1]$, respectively.

Lemma 7. Assume that $\kappa>0, b>c$, and $\lambda \neq 0$, if $v \in C\left([0,1] ; H^{3}\left(R^{2}\right)\right) \cap C^{1}\left([0,1] ; L^{2}\left(R^{2}\right)\right)$ is a function such that $v(t)$ is bounded in $H^{3}\left(e^{2 \kappa|x|} e^{2 \kappa|y|} d x d y\right)$ and $v^{\prime}(t) \in$ $L^{1}\left([0,1] ; L^{2}\left(e^{2 \kappa|x|} e^{2 \kappa|y|} d x d y\right)\right)$. Then,

$$
\begin{aligned}
& \left\|e^{\lambda x} v\right\|_{L^{2}\left(R^{2} \times[0,1]\right)} \\
& \leq\left\|e^{\lambda x} v(0)\right\|_{L^{2}\left(R^{2}\right)}+\left\|e^{\lambda x} v(1)\right\|_{L^{2}\left(R^{2}\right)} \\
& \quad+\left\|e^{\lambda x}\left(v_{t}+b v_{x x x}+c v_{y y x}+d v_{x}\right)\right\|_{L^{2}\left(R^{2} \times[0,1]\right)}, \\
& \left\|e^{\lambda y} v\right\|_{L^{2}\left(R^{2} \times[0,1]\right)} \\
& \leq\left\|e^{\lambda y} v(0)\right\|_{L^{2}\left(R^{2}\right)}+\left\|e^{\lambda y} v(1)\right\|_{L^{2}\left(R^{2}\right)} \\
& \quad+\left\|e^{\lambda y}\left(v_{t}+b v_{x x x}+c v_{y y x}+d v_{x}\right)\right\|_{L^{2}\left(R^{2} \times[0,1]\right)} .
\end{aligned}
$$

Proof. Let $p(t)=e^{\lambda x} v(t)$ and $q(t)=e^{\lambda x}\left(v_{t}+b v_{x x x}+c v_{y y x}+\right.$ $\left.d v_{x}\right)$; then,

$$
\begin{aligned}
q(t)= & e^{\lambda x} v^{\prime}(t)-\left(b \lambda^{3}+d \lambda\right) p(t)+\left(3 b \lambda^{2}+d\right) p_{x}(t) \\
& -3 b \lambda p_{x x}(t)+b p_{x x x}(t)-c \lambda p_{y y}(t)+c p_{y y x}(t) .
\end{aligned}
$$

Taking the spatial Fourier transform in (14) and applying Lemma 6, we have

$$
\frac{d}{d t}[p(t)]^{\wedge}(\xi)+[-i m(\xi)-n(\xi)][p(t)]^{\wedge}(\xi)=[q(t)]^{\wedge}(\xi),
$$

where

$$
\begin{gathered}
m(\xi)=-\left(3 b \lambda^{2}+d\right) \xi_{1}+b \xi_{1}^{3}+c \xi_{1} \xi_{2}^{2} \\
n(\xi)=\left(b \lambda^{3}+d \lambda\right)-3 b \lambda \xi_{1}^{2}-c \lambda \xi_{2}^{2} .
\end{gathered}
$$

According to (15), when $n(\xi) \leq 0$, we have

$$
\begin{aligned}
{[p(t)]^{\wedge}(\xi)=} & e^{i m(\xi) t} e^{n(\xi) t}[p(0)]^{\wedge}(\xi) \\
& +\int_{0}^{t} e^{i m(\xi)(t-\tau)} e^{n(\xi)(t-\tau)}[q(\tau)]^{\wedge}(\xi) d \tau,
\end{aligned}
$$

and when $n(\xi)>0$, we choose to write

$$
\begin{aligned}
{[p(t)]^{\wedge}(\xi)=} & e^{-i m(\xi)(1-t)} e^{-n(\xi)(1-t)}[p(1)]^{\wedge}(\xi) \\
& -\int_{t}^{1} e^{-i m(\xi)(\tau-t)} e^{-n(\xi)(\tau-t)}[q(\tau)]^{\wedge}(\xi) d \tau .
\end{aligned}
$$

Therefore, we have

$$
\begin{aligned}
\left|[p(t)]^{\wedge}(\xi)\right| \leq & \left|[p(0)]^{\wedge}(\xi)\right|+\left|[p(1)]^{\wedge}(\xi)\right| \\
& +\int_{0}^{1}\left|[q(\tau)]^{\wedge}(\xi)\right| d \tau, \quad t \in[0,1] .
\end{aligned}
$$

Appling Plancherel formula, we have inequality (12).

Similarly, letting $p(t)=e^{\lambda y} w(t)$, we can also have (13). This completes the proof of the lemma.

Lemma 8 (see $[12,13])$. Assume that $L>0$, and $b>c$, if $u \in C\left([0,1] ; H^{4}\left(R^{2}\right)\right)$ is a solution to (1) such that

$$
\operatorname{supp} u(t) \in[-L, L] \times[-L, L], \quad \forall t \in[0,1] ;
$$

then, $u \equiv 0$.

Proof. The proof is similar to that of Theorem 1.1 in [12], and we omit the details.

\section{Proof of the Main Result}

Assume that $\phi(x):=\widetilde{\phi}(x-A)$ for $A>L$ where $\widetilde{\phi}(x) \in C^{\infty}(R)$ is a nondecreasing function such that $\tilde{\phi}(x)=0$ for $x<0$ and $\widetilde{\phi}(x)=1$ for $x>1$. Let $g:=\phi(x) u$; then, $g(0)=g(1)=0$. According to Lemma 7, we obtain that

$$
\begin{aligned}
\left\|e^{\lambda x} w\right\| \leq & \left\|e^{\lambda x}\left(g_{t}+b g_{x x x}+c g_{y y x}+d g_{x}\right)\right\| \\
= & \| e^{\lambda x}\left[\phi u_{t}+b \phi u_{x x x}+c \phi u_{y y x}+\left(b \phi^{\prime \prime \prime}+d \phi^{\prime}\right) u\right. \\
& \left.+\left(3 b \phi^{\prime \prime}+d \phi\right) u_{x}+3 b \phi^{\prime} u_{x x}+c \phi^{\prime} u_{y y}\right] \| \\
\leq & \left\|a e^{\lambda x} \phi u u_{x}\right\|+\left\|e^{\lambda x} G_{\phi, u}\right\|,
\end{aligned}
$$

where $\|\cdot\|:=\|\cdot\|_{L^{2}\left(R^{2} \times[0,1]\right)}$ and

$$
G_{\phi, u}=\left(b \phi^{\prime \prime \prime}+d \phi^{\prime}\right) u+3 b \phi^{\prime \prime} u_{x}+3 b \phi^{\prime} u_{x x}+c \phi^{\prime} u_{y y} .
$$

Note that the derivatives of $\phi(x)$ are supported in the interval $[A, A+1]$; then,

$$
\left\|e^{\lambda x} G_{\phi, u}\right\|=C e^{\lambda(A+1)},
$$

where $C$ is dependent on $\|u\|_{C\left([0,1] ; H^{2}\right)}$ and $\max \{b, c, d\}$.

Combining (21) with (23), we obtain

$$
\left\|e^{\lambda x} \phi u\right\| \leq a\left\|e^{\lambda x} \phi u\right\|\left\|u_{x}\right\|_{L^{\infty}([A,+\infty] \times R \times[0,1])}+C e^{\lambda(A+1)} .
$$

Applying Lemma 4 with $\kappa=1$, we have that

$$
\left|u_{x}(t)(x, y)\right| \leq C_{1} e^{-x},
$$

then

$$
\left\|e^{\lambda x} \phi u\right\| \leq a C_{1} e^{-A}\left\|e^{\lambda x} \phi u\right\|+C e^{\lambda(A+1)} .
$$


Since $\left\|e^{\lambda x} \phi u\right\|<+\infty$, taking $A>\max \{L, 1\}$ such that $C_{1} e^{-A}$ $<1 / 2 a$, we have

$$
\left\|e^{\lambda x} \phi u\right\| \leq C e^{\lambda(A+1)} .
$$

Note that $\phi(x)=1$ for $x \geq 2 A$; we have

$$
e^{2 \lambda A}\left(\int_{0}^{1} \int_{-\infty}^{\infty} \int_{2 A}^{\infty}|u(t)(x, y)|^{2}\right)^{1 / 2} \leq\left\|e^{\lambda x} \phi u\right\| \leq C e^{\lambda(A+1)} .
$$

Letting $\lambda \rightarrow+\infty$, we obtain

$$
\left(\int_{0}^{1} \int_{-\infty}^{\infty} \int_{2 A}^{\infty}|u(t)(x, y)|^{2}\right)^{1 / 2}=0
$$

and this proves that $u \equiv 0$ in $[2 A, \infty) \times R \times[0,1]$.

Next, we will prove that $u \equiv 0$ in $R \times[2 A, \infty) \times[0,1]$. Let $h=\phi(y) u$; then,

$$
\begin{aligned}
\left\|e^{\lambda y} h\right\| \leq & \left\|e^{\lambda y}\left(h_{t}+b h_{x x x}+c h_{y y x}+d h_{x}\right)\right\| \\
= & \| e^{\lambda y}\left(\phi u_{t}+b \phi u_{x x x}+c \phi u_{y y x}+d \phi u_{x}+2 c \phi^{\prime} u_{x y}\right. \\
& \left.+c \phi^{\prime \prime} u_{x}\right) \| \\
& \leq\left\|a e^{\lambda y} \phi u u_{x}\right\|+\left\|e^{\lambda y} H_{\phi, u}\right\|,
\end{aligned}
$$

where $H_{\phi, u}:=2 c \phi^{\prime} u_{x y}+c \phi^{\prime \prime} u_{x}$.

In fact, Let $\tilde{u}(x, y, t)=u(-x,-y, 1-t)$; it is easy to check that $\tilde{u}$ also satisfies the hypotheses of this theorem, and then we find that $u \equiv 0$ in

$$
(-\infty,-2 A] \times R \times[0,1] \cup R \times(-\infty,-2 A] \times[0,1] .
$$

Thus, there exists $A>0$ such that $\operatorname{suppu}(t) \in[-A, A] \times$ $[-A, A]$ for all $t \in[0,1]$. Applying Lemma 8 , we complete the proof of Theorem 1 .

\section{Acknowledgments}

This work was supported by the National Natural Science Foundation of China (no. 11171135), the Natural Science Foundation of Jiangsu (no. BK 2010329), the Project of Excellent Discipline Construction of Jiangsu Province of China, the Priority Academic Program Development of Jiangsu Higher Education Institutions, and the Natural Science Foundation of the Jiangsu Higher Education Institutions of China (no. 09KJB110003), as well as the Taizhou Social Development Project (no. 2011213).

\section{References}

[1] G. A. Gottwald, "The Zakharov-Kuznetsov equation as a twodimensional model for nonlinear Rossby wave," http://arxiv .org/abs/nlin/0312009.

[2] J. H. He, "Application of homotopy perturbation method to nonlinear wave equations," Chaos, Solitons and Fractals, vol. 26, no. 3, pp. 695-700, 2005.
[3] Z. Fu, S. Liu, and S. Liu, "Multiple structures of two-dimensional nonlinear Rossby wave," Chaos, Solitons and Fractals, vol. 24, no. 1, pp. 383-390, 2005.

[4] A. A. Aslan, "Generalized solitary and periodic wave solutions to a (2+1)-dimensional Zakharov-Kuznetsov equation," Applied Mathematics and Computation, vol. 217, no. 4, pp. 1421-1429, 2010.

[5] V. E. Zakharov and E. A. Kuznetsov, "On three-dimensional solitons," Journal of Experimental and Theoretical Physics, vol. 39, pp. 285-286, 1974.

[6] B. K. Shivamoggi, "The Painlevé analysis of the ZakharovKuznetsov equation," Physica Scripta, vol. 42, no. 6, pp. 641-642, 1990.

[7] F. Linares and A. Pastor, "Local and global well-posedness for the 2D generalized Zakharov-Kuznetsov equation," Journal of Functional Analysis, vol. 260, no. 4, pp. 1060-1085, 2011.

[8] A. V. Faminskiî, "The Cauchy problem for the ZakharovKuznetsov equation," Differential Equations, vol. 31, no. 6, pp. 1002-1012, 1995.

[9] H. A. Biagioni and F. Linares, "Well-posedness results for the modified Zakharov-Kuznetsov equation," in Nonlinear Equations: Methods, Models and Applications, vol. 54 of Progress in Nonlinear Differential Equations and Their Applications, pp. 181189, Birkhäuser, Basel, Switzerland, 2003.

[10] F. Linares and A. Pastor, "Well-posedness for the twodimensional modified Zakharov-Kuznetsov equation," SIAM Journal on Mathematical Analysis, vol. 41, no. 4, pp. 1323-1339, 2009.

[11] F. Linares, A. Pastor, and J.-C. Saut, "Well-posedness for the ZK equation in a cylinder and on the background of a KdV soliton," Communications in Partial Differential Equations, vol. 35, no. 9, pp. 1674-1689, 2010.

[12] M. Panthee, "A note on the unique continuation property for Zakharov-Kuznetsov equation," Nonlinear Analysis. Theory, Methods \& Applications, vol. 59, no. 3, pp. 425-438, 2004.

[13] E. Bustamante, P. Isaza, and J. Mejía, "On the support of solutions to the Zakharov-Kuznetsov equation," Journal of Differential Equations, vol. 251, no. 10, pp. 2728-2736, 2011.

[14] J. Bourgain, "On the compactness of the support of solutions of dispersive equations," International Mathematics Research Notices, no. 9, pp. 437-447, 1997.

[15] C. E. Kenig, G. Ponce, and L. Vega, "On the support of solutions to the generalized KdV equation," Annales de l'Institut Henri Poincaré. Analyse Non Linéaire, vol. 19, no. 2, pp. 191-208, 2002. 


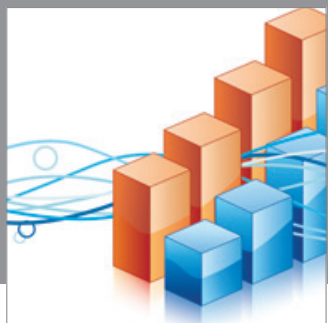

Advances in

Operations Research

mansans

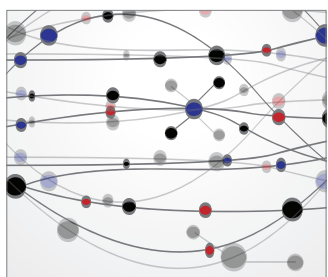

The Scientific World Journal
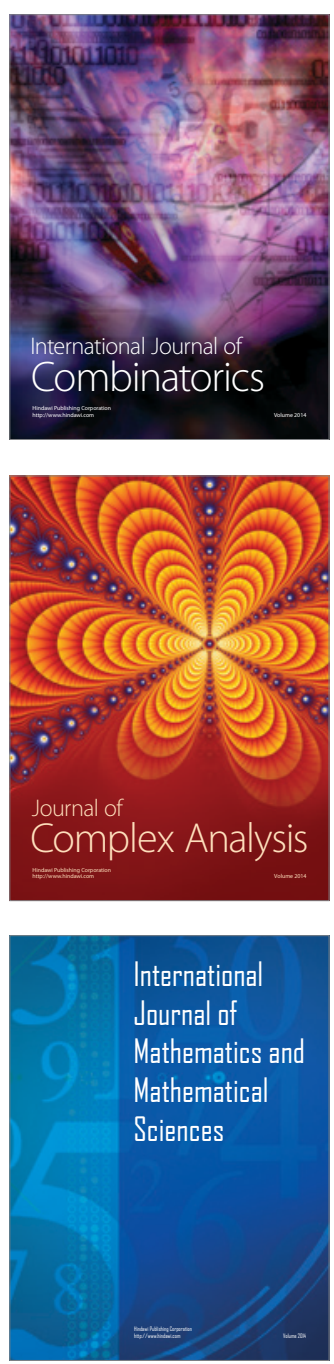
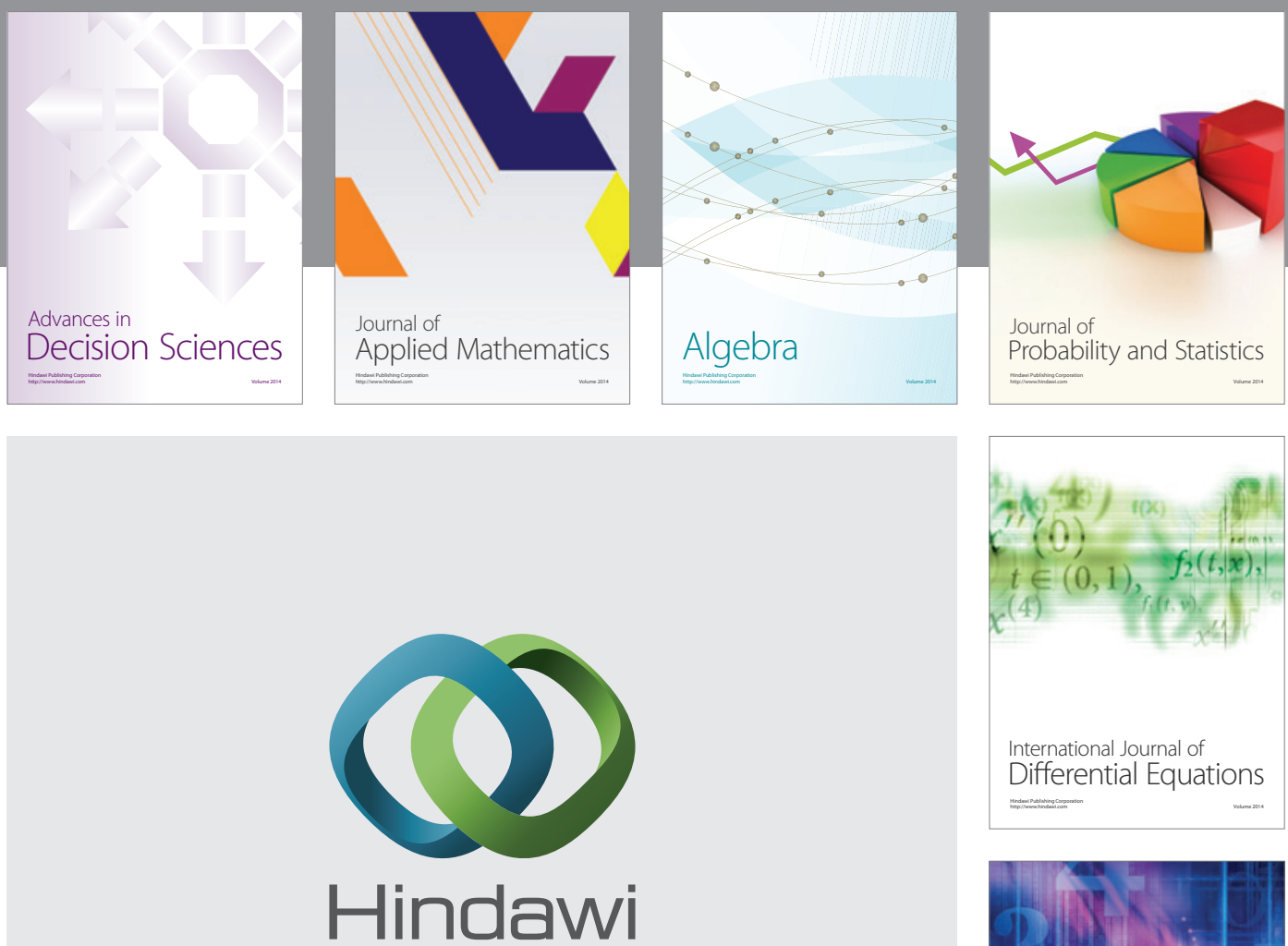

Submit your manuscripts at http://www.hindawi.com
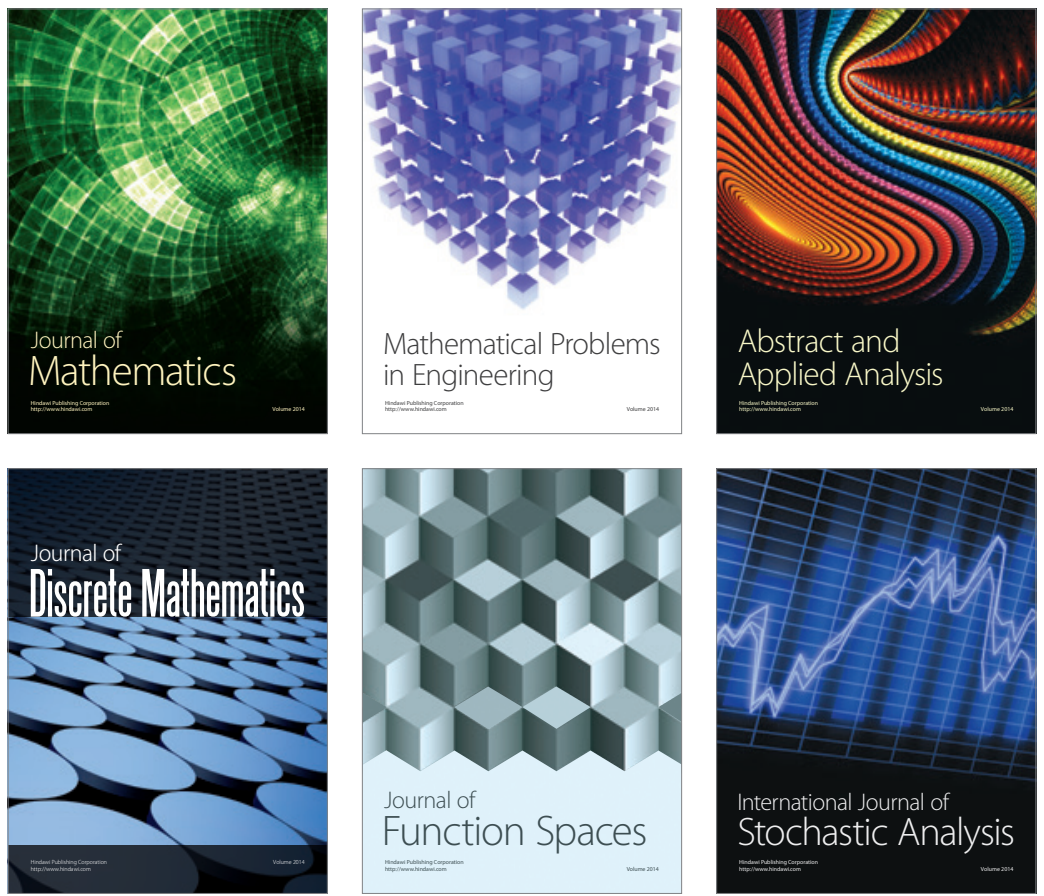

Journal of

Function Spaces

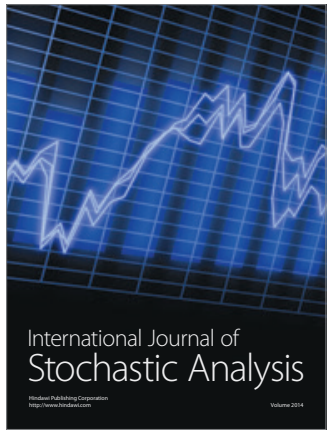

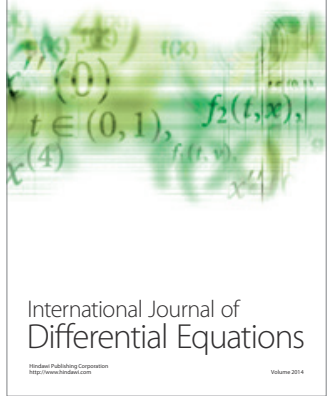
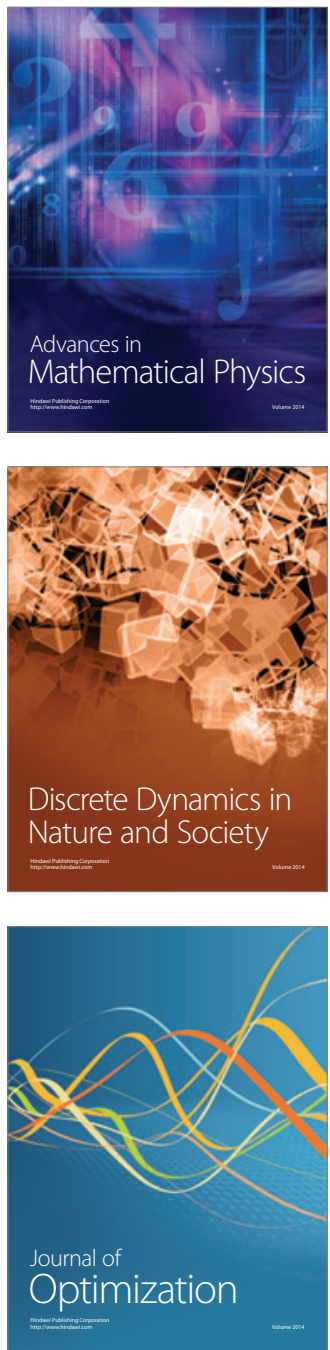